\title{
Propagation of localized, unsteady heat loads in aircraft cabin air flows
}

\author{
J. Bosbach ${ }^{1}$ T. Dehne ${ }^{1}$
}

Received: 16 December 2014/Revised: 8 October 2015/Accepted: 20 October 2015/Published online: 3 November 2015

(C) Deutsches Zentrum für Luft- und Raumfahrt e.V. 2015

\begin{abstract}
We studied experimentally the propagation of heat, released from a local source, in aircraft cabin air flow using two different ventilation systems. Besides the stateof-the-art mixing ventilation system, a ceiling-based cabin displacement ventilation system was employed. As test environment we used the Do 728 test facility of the German Aerospace Center in Göttingen. To measure the response of the cabin temperatures to the released heat, we evaluated the cross-correlation function between the local temperatures and the normalized surface temperature of the heat source. The latter was heated periodically at three different nominal heating powers, ranging from $100 \mathrm{~W}$ through $400 \mathrm{~W}$. By analyzing the data, we could observe the gradual change of the temperature from a passive to an active scalar. While the two ventilation systems reveal a similar behavior at the lower source powers, the distinguished air-guiding principles of the momentum-driven mixing ventilation and the buoyancy-driven ceiling-based cabin displacement ventilation imply different propagation paths at higher power settings of the heat source. For the first time, the spatial spreading of locally released heat in mixing ventilation and in ceiling-based displacement ventilation was determined in an aircraft cabin.
\end{abstract}

This paper is based on a presentation at the German Aerospace Congress, September 16-18, 2014, Augsburg, Germany.

\section{J. Bosbach}

Johannes.Bosbach@dlr.de

1 German Aerospace Center (DLR), Institute of Aerodynamics and Flow Technology, Bunsenstr. 10, 37073 Göttingen, Germany
Keywords Indoor air flow - Mixing ventilation . Displacement ventilation - Mixed convection . Heat propagation $\cdot$ Active scalar

\section{Introduction}

The general trend of increasing heat loads in modern passenger aircraft cabins during the last years [1] induced a demand for efficient yet comfortable ventilation concepts. While the steady performance of state-of-the-art and novel ventilation concepts has been studied extensively (see e.g. [2-7]), only few studies address their dynamic performance and stability with respect to non-ideal boundary conditions. Besides the static performance of such systems, however, the dynamic behavior and stability with respect to operational boundary conditions is crucial. Since established and alternative ventilation concepts rely upon different physical forces to generate their characteristic global flow patterns, their response to non-ideal and time-dependent boundary conditions is expected to be well distinguished. The number of effects, which might influence the performance of cabin ventilation, is extensive and comprises temporal variation of heat loads (in-flight entertainment, galleys), asymmetrically distributed heat loads (solar radiation, incompletely occupied cabin), jet-jet interaction, jetplume interaction and moving passengers (obstruction of and interaction with jets, wake generation).

Our study aims to approach this complex issue by investigating the propagation of localized, time varying heat loads in a full-scale experiment in the Do 728 aircraft cabin test facility of the German Aerospace Center. The selected test environment provides three different ventilation systems, i.e. mixing ventilation (MV), cabin displacement ventilation (CDV) and ceiling-based cabin 
displacement ventilation (CCDV), which can be used solely or combined [7]. A remotely controllable heat source with a fast time response has been developed and used in the experiments. Releasing heat periodically, the response of the cabin air flow has been monitored with a sensor installation, which comprises more than 340 probes and 70 thermal passenger dummies. As a result, the spatial spreading and propagation dynamics of the released heat was characterized for different ventilation systems.

\section{Transient effects in ventilation of aircraft cabins}

Acquisition of dynamic effects in turbulent aircraft cabin ventilation states a challenge due to the non-stationary nature of the flow and the large range of involved time scales. Beyond the turbulent energy cascade of the flow itself, the most important time scale is given by the nominal air exchange time of the cabin, which is of the order of $10^{2} \mathrm{~s}$. In addition, the thermal diffusion times of the cabin interior parts, which amount to about $10^{3} \mathrm{~s}$, are incorporated. In many cases, the effects to be studied may be superimposed by the non-stationary nature of the flow, requiring appropriate averaging over many cycles. Consequently, the amount of literature on transient effects in aircraft cabin ventilation is by far smaller as compared to the steady performance. Nevertheless, an improved understanding of such effects is mandatory for e.g. a better prediction of transmission paths of infectious diseases [8]. The latter are expectedly restricted by the ventilation system and thus determined by the applied ventilation concept.

In mixing ventilation, the structure and dynamics of the large scale flow structures are governed by jet-jet interaction, negative buoyancy forces and jet-plume interaction $[2,9]$. Instabilities in these systems are able to even induce global changes of the large scale air circulations [9]. During operational conditions, such settings should be of course avoided to guarantee the thermal comfort and air quality throughout the whole flight.

Typical transient scenario under operational conditions are cabin "pull-down" and "pull-up", where a warm cabin has to be cooled down in a short time and vice versa. For a cabin displacement ventilation (CDV) as well as a hybrid cabin displacement and mixing ventilation system (HV), the "pull-up" and "pull-down" performance was experimentally quantified in an A320 aircraft cabin under flight conditions $[5,10]$. It was found, that both systems are able to increase or reduce the cabin air temperature by $3 \mathrm{~K}$ within $<11$ min under flight conditions. While CDV performed better for the cabin air temperatures, HV, which provides higher turbulence levels in the cabin flow, was able to cool the surfaces faster.
Related to this topic is a study by Bianco et al. [11], who numerically simulated transient temperatures and flow velocities in an executive aircraft cabin section at MV. The thermal coupling of the cabin interior to the external environment and fuselage was considered using a global thermal conductance. However, the simulated cabin was completely empty. Bianco et al. [11] thus state that the corresponding results have only limited practical application.

An issue very similar to the question addressed in our study is the contaminant transport in aircraft cabin air flow, which was e.g. studied experimentally and numerically by Yan et al. [12]. Tracer gas experiments with $\mathrm{CO}_{2}$ were conducted in a full-scale Boeing 767-300 mock-up and supported by CFD simulations under isothermal conditions. Iso-concentration contours and temporal concentration evolutions were determined and a strong dependence on the $\mathrm{CO}_{2}$ release position was observed. However, propagation of pollutants was restricted to about plus minus one to two seat rows in flight direction but extended up to the whole seat row.

While understanding of pollutants propagation in a real cabin geometry at mixing ventilation is already demanding, operational conditions further involve standing or moving bodies in the aisle region. The latter will generate wakes, in which pollutants might be transported much farther than by pure MV.

Studies of contaminant transport have been conducted using a small-scale, isothermal water model and validated CFD simulations by Mazdumar et al. [13]. They found that the seats and passengers tended to obstruct the lateral transport of the contaminants and confined their spread to the aisle of the cabin. However, a moving person in the aisle could, according to Mazdumar et al. [13], carry a contaminant in its wake to as many rows as the person passed. They conclude, that this could be the reason why a passenger infected with the severe acute respiratory syndrome (SARS) could infect fellow passengers who were seated seven rows away [8]. However, the result was depending on the shape of the moving body, which should be a human-like model for accurate simulation of the contaminant transport [13].

The impact of a periodically unsteady air supply on the transient flow fields in an aircraft cabin was studied numerically by $\mathrm{Wu}$ et al. [14]. They found that the averaged temperature and $\mathrm{CO}_{2}$ concentration levels could be reduced within the whole domain and in typical breathing zones compared to the conventional steady-supply using a periodic air supply. The improvement was brought about by a dynamic change of the jet paths, which resulted in an extension of the regions with high local air exchange and a reduction of regions with still water. 
To our knowledge, there are currently no studies available, which address the propagation of localized heat loads in aircraft cabin air flows.

\section{Aircraft and test installation}

\subsection{General description}

We used the Do 728 test facility of the German Aerospace Center in Göttingen, which is a grounded test facility and predicated on the test aircraft 728 No. 1 of Fairchild Dornier, as test environment. It provides a realistic and sound cabin structure of a short-range aircraft cabin. Figure 1 depicts a cross section and Fig. 2 a top view of the cabin, which has a total length of $16.9 \mathrm{~m}$, a width of $3.25 \mathrm{~m}$ and a height of $2.14 \mathrm{~m}$. An external heating, ventilation and air conditioning (HVAC) system is used for air supply and extraction.
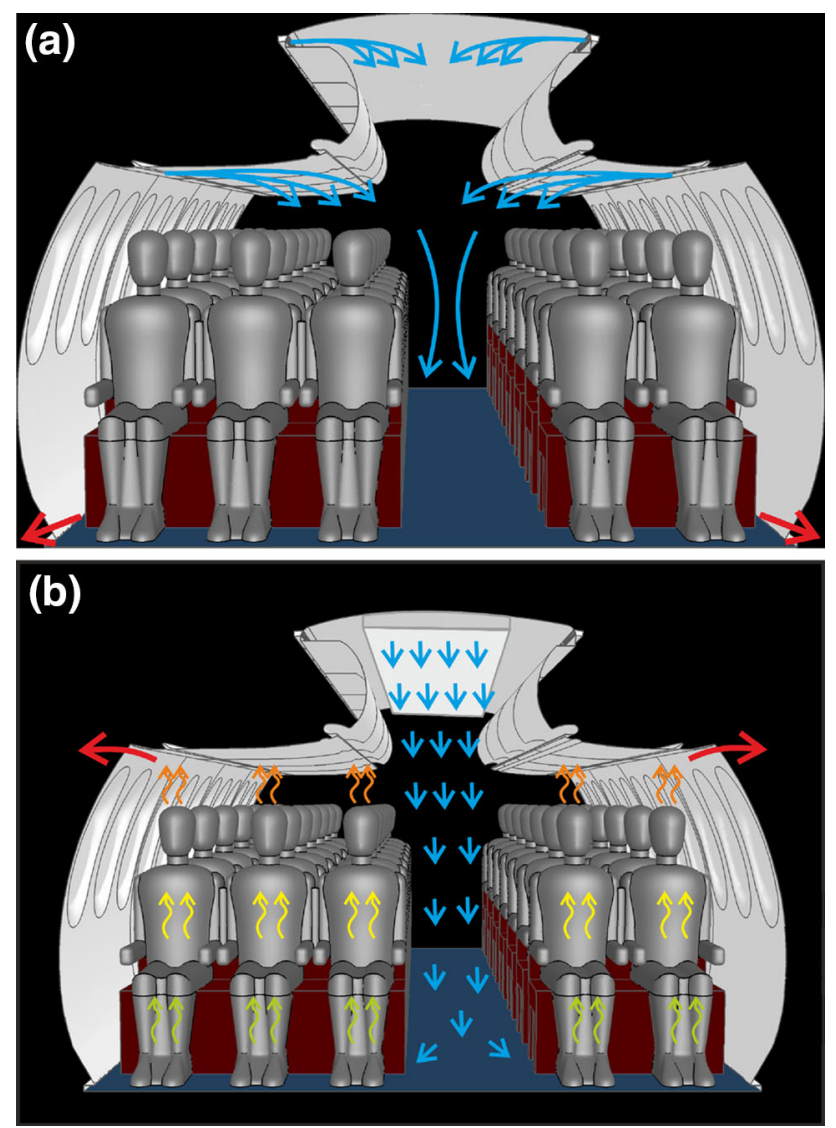

Fig. 1 Illustration of the investigated ventilation scenarios, reproduced from [7]. a Mixing ventilation (MV) b ceiling-based cabin displacement ventilation (CCDV). For further explanations, see text

\subsection{Ventilation systems and cabin layout}

Currently, three different ventilation systems are installed in the Do 728. Besides the original MV system, a cabin displacement ventilation (CDV) system and a ceiling-based cabin displacement ventilation (CCDV) system have been integrated (for a detailed description see [7]). Combining these different systems, a variety of hybrid ventilation scenarios can be realized.

With the MV system, fresh air is supplied by the lateral and ceiling cabin air outlets on both sides of the cabin; see Fig. 1a. The air is injected with rather high momentum into the cabin-typical entrance velocities amount to about $1 \mathrm{~m} / \mathrm{s}$. As a consequence, air jets are generated, which follow the adjacent contours due to the Coanda effect. The stress in the shear layers of these jets causes an efficient mixing of fresh and recirculated air, while the different jets organize into large scale roll structures. Finally, the recirculated air leaves the cabin below the dado panels. In our case, the air is removed by active suction through air outlets behind the dado panels.

The other scenario, which is addressed here, is depicted in Fig. 1b. Fresh air is supplied with very small momentum through the CCDV outlets. The outlet velocities are well below $0.1 \mathrm{~m} / \mathrm{s}$. As a consequence, an unstable thermal stratification is induced, which breaks up into, small and intermediate scale flow structures. The latter finally organize into the large scale flow structures. After circulation in the cabin, the air leaves through the inversely operated lateral MV outlets.

An important difference between MV and CCDV relies in the fact, that at MV, the flow structures are imprinted by the momentum of the air jets, and thus far more kinetic energy is brought into the cabin. At CCDV, on the other hand, with the fresh air, mainly a temperature gradient is induced in the cabin, and the flow structures are a result of these.

\subsection{Investigated ventilation scenarios}

The investigated ventilation scenarios were operated at a nominal volume flow rate of $600 \mathrm{l} / \mathrm{s}$, which corresponds to a mass flow rate of $0.742 \mathrm{~kg} / \mathrm{s}$ or a volume flow rate of $574 \mathrm{l} / \mathrm{s}$ at standard conditions. The nominal time required for a full air exchange, $\tau_{\mathrm{ex}}$, amounted to about $90 \mathrm{~s}$. In order to address the asymmetric cabin layout, $3 / 5$ of the total volume flow rate were supplied and removed via the in- and outlets, respectively, on the right cabin side (in flight-direction) and 2/5 from the left cabin side. At MV, $50 \%$ of the volume flow rate was provided by the lateral and $50 \%$ by the ceiling outlets.

The measurement section (MS) was separated by two polystyrene walls with a thickness of $0.1 \mathrm{~m}$ each in order 


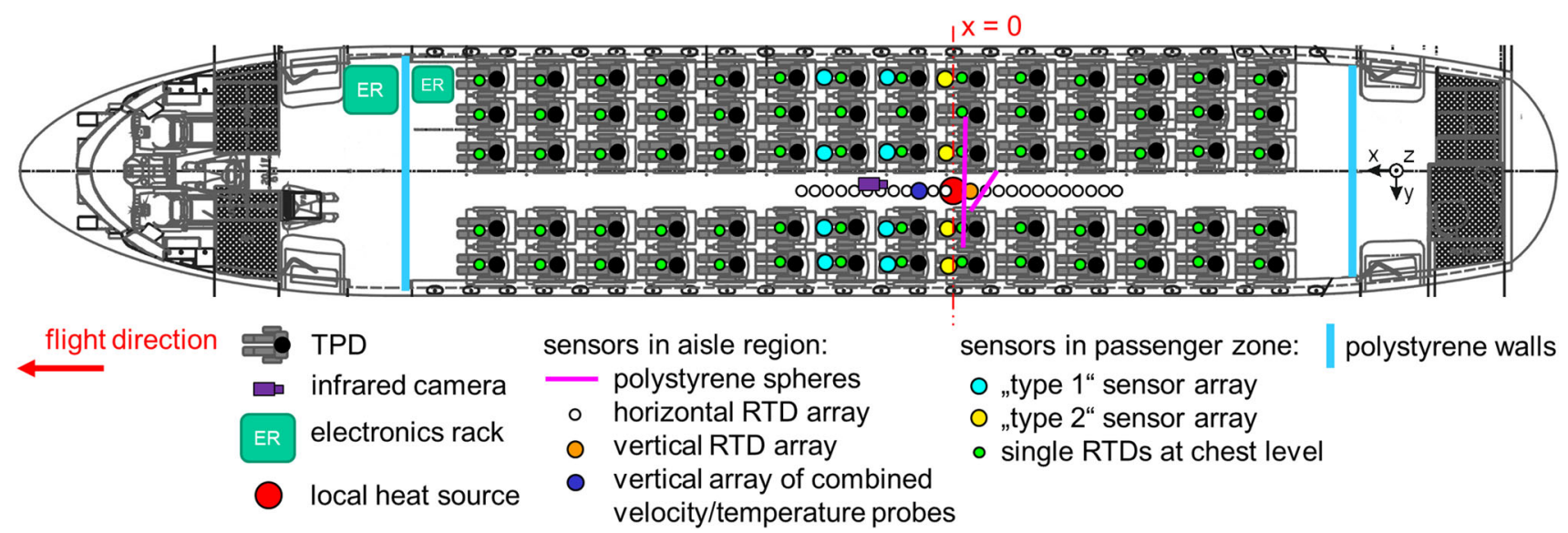

Fig. 2 Cabin layout and measurement installation in the Do 728

to minimize boundary effects. 70 thermal passenger dummies (TPDs) were placed inside the MS at a seat pitch of $33^{\prime \prime}$, see Fig. 2. They provide a total thermal impact of $5.25 \mathrm{~kW}$. The electronics rack of the cabin test installation and the cabin illumination caused an additional heat load of 37 and $620 \mathrm{~W}$ in the MS, respectively. The power released by the infrared camera amounted to $<23 \mathrm{~W}$. Prior to the actual measurements, the whole cabin was allowed to equilibrate for several hours with the ventilation and TPDs operating until stationary thermal conditions were achieved.

\section{Measurement installation}

\subsection{General description}

A cabin measurement installation comprising 70 TPDs and more than 340 local temperature and velocity sensors was installed in the passenger zone and the aisle in order to generate and capture a realistic cabin environment.

\subsection{Thermal passenger dummies}

To ensure realistic heat loads and obstructions, which are important for generation of buoyancy forces and dissipation of momentum, TPDs were placed on the seats in the passenger zone. The TPDs were designed for experimental simulation of indoor airflow, and their main benefit is, that they exhibit a very homogeneous heat flux density, which is slightly increased on purpose in the head region. A homogeneous distribution of the heat load over the TPD surface is a necessity in order to accomplish realistic surface temperatures and thus buoyancy forces. They were operated at a constant heat flux of $75 \mathrm{~W}$; each has a volume of $0.05 \mathrm{~m}^{3}$ and a surface of $1.5 \mathrm{~m}^{2}$.

\subsection{Local heat source}

The local heat source employed in the experiments was designed to provide an instantaneous and constant heat release. Consequently, our design aimed at minimization of the thermal inertia of the source. Due to its high degree of symmetry, we chose a cylindrical geometry, see Fig. 3. While the top and bottom plates are made of acrylic glass plates with a thickness of $5 \mathrm{~mm}$, the sidewall consists of steel gauze with a mesh width of $0.25 \mathrm{~mm}$ and a wire diameter of $0.1 \mathrm{~mm}$. The top and bottom end of the gauze sleeve was electrically connected via a ring electrode to a programmable power supply. Thus, the active surface of the heat load, which amounts to $0.68 \mathrm{~m}^{2}$, could be heated with a peak power of up to $1.2 \mathrm{~kW}$. The temperature response of the source was monitored with five resistance temperature detectors (RTD), connected to the gauze at four heights $(0.1,0.25,0.4,0.55$ and $0.7 \mathrm{~m}$ of the gauze sleeve), see Fig. 3. During the measurements, the heat source was switched on and off periodically at a period of $600 \mathrm{~s}$. Duty and idle cycle amounted to $300 \mathrm{~s}$ each.

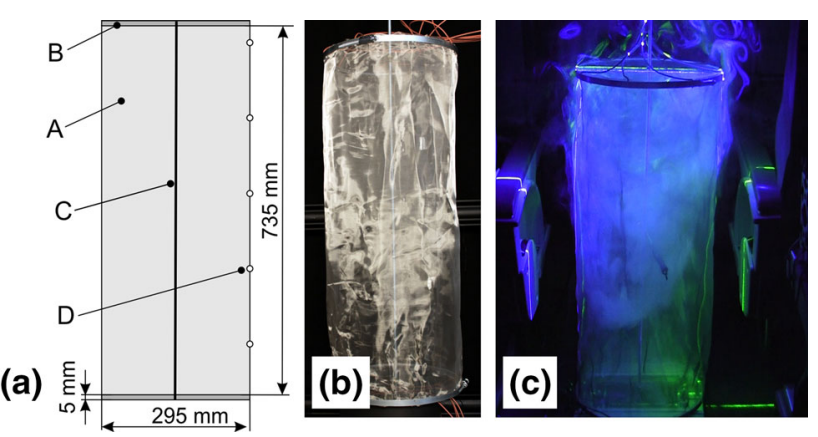

Fig. 3 Local heat source a sketch with the main dimensions and components ( $A$ steel gauze, $B$ top and bottom plate, $C$ threaded rod, $D$ RTDs) b photo c smoke visualization at free thermal convection 
During the duty cycle, the source was operated at three different nominal powers $P_{\mathrm{H}}$ of 100,200 and $400 \mathrm{~W}$. While the two lower settings were meant to resemble the heat load generated by one and two moving persons in the aisle, the high power setting was employed to explore the limiting behavior of the ventilation scenarios. In order to achieve an almost instantaneous heat release during the duty cycle, the electrical power was adjusted in three steps during the duty cycle with the aim to overcome the thermal inertia of the heat source itself. Since the excess heat was released during the idle cycle, the mean power during the full period, $\langle P\rangle$, was increased by up to $10 \%$. The values used for the different nominal heating powers are summarized in Table 1. For the measurements described in the following, the source was positioned in the aisle at the height of the 9th seat row, see Fig. 2. As an example, the temperature response of the heat source to the applied power is plotted in Fig. 4 for CCDV at a source power of $200 \mathrm{~W}$. A strong temperature stratification can be detected, which is induced by the source itself. While the rising edge of the temperatures is very steep, the falling edge is limited by the thermal inertia of the source and slowed down accordingly. Depending on the actual settings and the ventilation scenario, the rise time of the source amounts to $<30 \mathrm{~s}$ for reaching $90 \%$ of the final temperature jump. Our actual experiments spanned typically 18 cycles of the heat source each, with a minimum of 16 periods.

\subsection{Sensor installation}

The basic cabin sensor installation of the Do 728 , which is described in [7], was extended for the current investigation. While the basic installation already provides temperature and velocity sensors on several levels in the center of the passenger zone and in the aisle as well as surface temperature sensors, further probes have been integrated. To obtain an overview of the heat propagation in the cabin, RTDs have been installed at chest level $(z=880 \mathrm{~mm})$ at a distance of $50 \mathrm{~mm}$ in front of each TPD. A horizontal RTD array with RTDs at a sensor spacing of $0.19 \mathrm{~m}$ in front and back (in flight direction) of the heat source has been installed in the aisle, see Fig. 5. Further, a vertical RTD array with sensors at 11 levels was positioned $0.06 \mathrm{~m}$ behind the heat source in the aisle, see Fig. 5.

Table 1 Power settings for the local heat source during the experiments

\begin{tabular}{|c|c|c|c|c|c|}
\hline \multirow{2}{*}{$\frac{\mathrm{P}_{\mathrm{H}}(\mathrm{W})}{100}$} & \multicolumn{4}{|c|}{ Power (W)/duration (s) } & \multirow{2}{*}{$\frac{\langle P\rangle(\mathrm{W})}{54}$} \\
\hline & $500 / 5$ & $110 / 15$ & $100 / 280$ & $0 / 300$ & \\
\hline 200 & $1000 / 5$ & $220 / 30$ & $200 / 265$ & $0 / 300$ & 108 \\
\hline 400 & $1200 / 12$ & $440 / 60$ & $400 / 228$ & $0 / 300$ & 220 \\
\hline
\end{tabular}

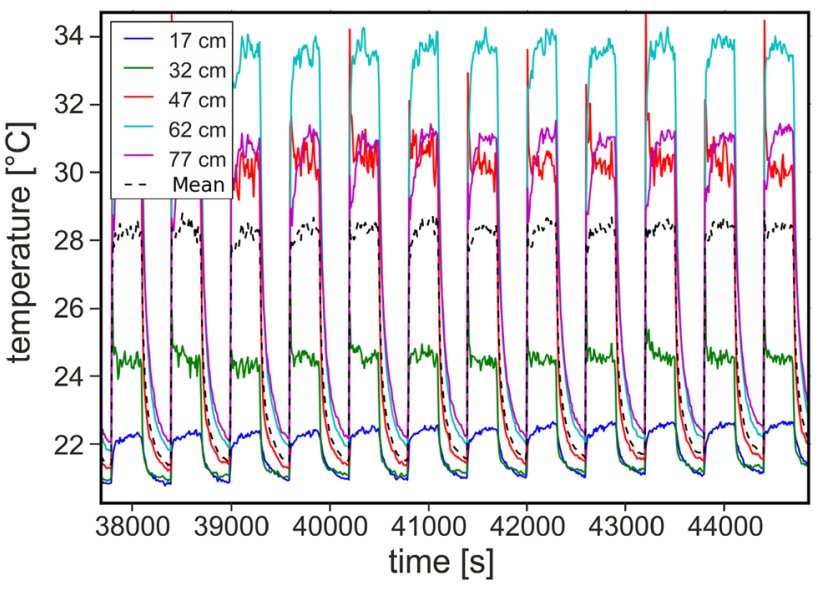

Fig. 4 Surface temperatures of the heat source measured with the RTDs depicted in Fig. 3 and mean for CCDV at $P_{\mathrm{H}}=200 \mathrm{~W}$. The values in the legend denote the height of the sensors relative to the cabin floor

To measure the temperatures of the air flowing into and out of the cabin, 96 RTDs were used ( 28 for the lateral MV outlets, 28 for the extraction behind the dado panels, 16 for the ceiling MV outlets, 16 for the CDV outlets and 8 for the CCDV outlets). The employed RTDs are of the type Pt100 (1/3 DIN B) and connected to a Keithley 3706 high precision multimeter via four wire circuits. Due to the high precision of the electronic circuitry, the main uncertainty for the RTDs is the calibration accuracy, which is below $0.15 \mathrm{~K}$ for the absolute temperature of a single sensor in our temperature range. The sampling rate of the RTDs amounted to $0.1 \mathrm{~Hz}$. An infrared camera was installed in the aisle in combination with a grid of polystyrene spheres to simultaneously capture surface and fluid temperatures. However, the results of these measurements are not subject of the current article.

\section{Methodology}

As an example, the temperature response to the periodic heat load at some positions in the passenger zone and at the surface of the local heat source are depicted in Fig. 6 for $\operatorname{CCDV}\left(P_{\mathrm{H}}=200 \mathrm{~W}\right)$. Clearly, the fluctuations of the temperature signals unveil the turbulent nature of the cabin flow. Herewith, the turbulent temperature fluctuations are similar in strength as the temperature fluctuations caused by our heat source. Nevertheless, the periodic response of the sensors is still visible in the plots. In order to improve the signal to noise ratio for further evaluation, we evaluated the cross-correlation between the sensor and reference signals:

$\mathrm{CCF}=\frac{\int R(t) \times S(t+\tau) \mathrm{dt}}{\int R(t) \times R(t) \mathrm{d} t}=\frac{(R \star S)(\tau)}{(R \star R)(0)}$ 
Fig. 5 Arrangement of the horizontal and vertical RTD arrays in the aisle with respect to the heat source. The combined velocity/temperature probe vertical array would be visible as well in this view but is not shown for the sake of clarity. A ceiling contour for CCDV outlets installed, $B$ ceiling contour for MV

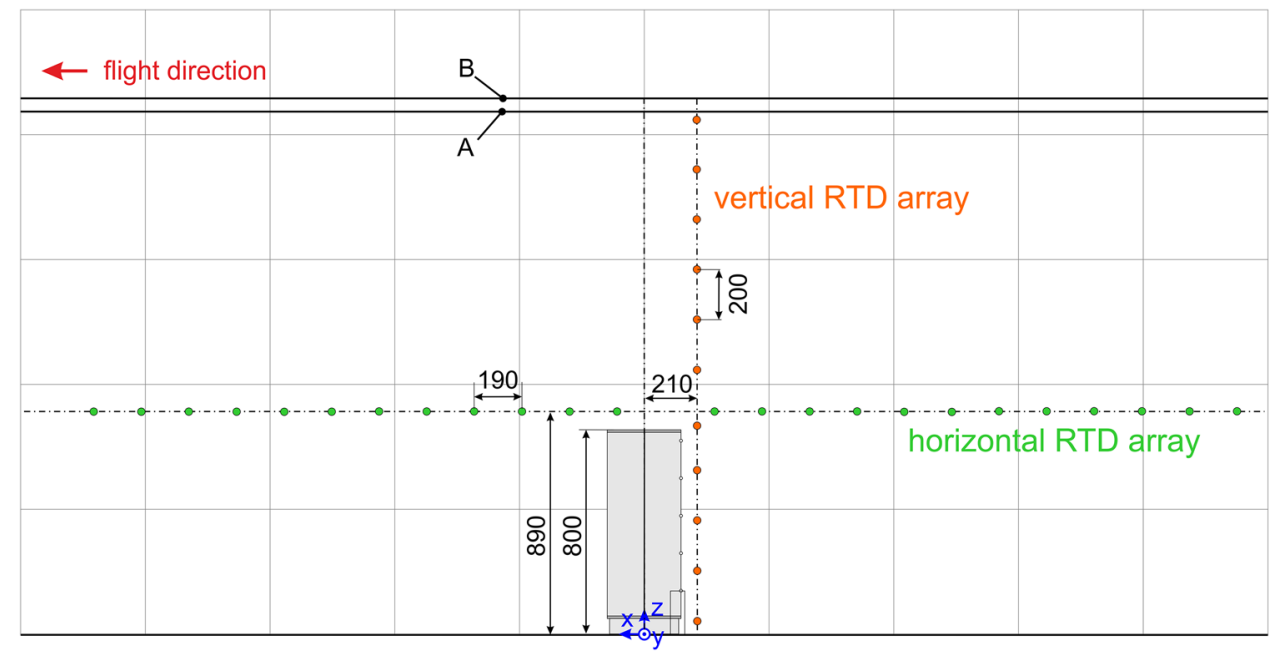

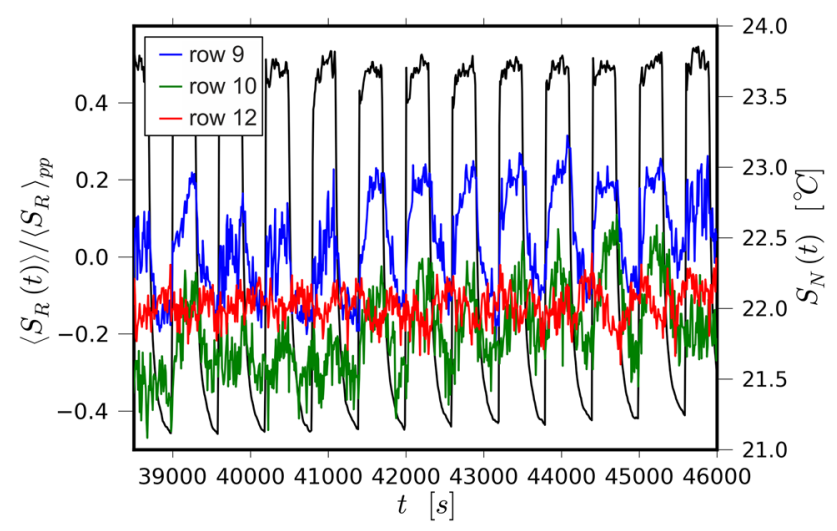

Fig. 6 Normalized mean surface temperature of the heat source (black) and fluid temperatures at three selected sensor positions for CCDV and a source power of $200 \mathrm{~W}$. The sensors are located at chest height at the aisle seats on the right hand cabin side in the designated rows

Herewith, $S(t)$ denotes the respective sensor signal and $R(t)$ a reference signal, which scales linearly with the heat released by the source. Prior to calculation of the crosscorrelation, the mean temperature drift has been removed by subtracting a least squares fit of a second- and first degree polynomial from signal and reference, respectively. As the turbulent temperature fluctuations between different sensor positions are uncorrelated, this approach allows to quantify the response of the local temperatures to the heat load under elimination of the turbulent temperature fluctuations. The reference signal was deduced from the average of the temperature sensors at the surface of the heat source:

$R(t)=\frac{\left\langle S_{\mathrm{R}}(t)\right\rangle}{\left\langle S_{\mathrm{R}}\right\rangle_{\mathrm{pp}}} \times \frac{P_{H}^{m}}{q_{M} c_{p}}$

Herewith, $\left\langle S_{\mathrm{R}}(t)\right\rangle$ denotes the spatial mean of the reference sensor signals, $\left\langle S_{\mathrm{R}}\right\rangle_{\mathrm{pp}}$ the peak to peak value of $\left\langle S_{\mathrm{R}}(t)\right\rangle, P_{H}^{m}$ the measured mean heat flux of the heat source during the main period of the heating cycle in Table $1, q_{M}$ the mass flow rate of the cabin and $c_{\mathrm{p}}$ the specific heat capacity of air. As a result to this definition of the reference signal, CCF is normalized to the power of the heat source and the inverse mass flow of the cabin. The correlation functions for the sensor signals shown in Fig. 6 are depicted in Fig. 7. It can be observed, that both, amplitude and phase, of the local temperature responses depend on the measurement position. However, in the following, we will focus on the magnitude of the first extremum of the cross-correlation functions, when moving backward in time, $\mathrm{CCF}_{\text {ex }}$, see Fig. 7 .

According to the chosen normalization, the local temperature response to the heat load can be calculated from the $\mathrm{CCF}_{\mathrm{ex}}$ value as follows:

$\Delta S=\mathrm{CCF}_{\mathrm{ex}} \frac{P_{H}^{m}}{q_{M} c_{p}}$

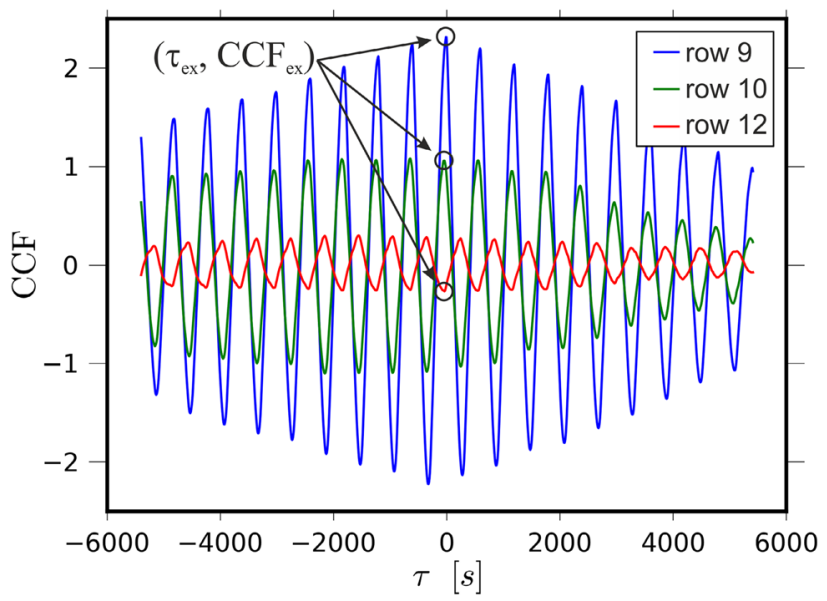

Fig. 7 Cross correlation functions of the sensor signals shown in Fig. 6 with the reference signal, for details see text 
Obviously, the meaning of $\mathrm{CCF}_{\mathrm{ex}}$ is the local temperature response to the heat load as compared to a complete dilution of the heat in the volume flow of the cabin. It should be noted, though, that $\mathrm{CCF}_{\mathrm{ex}}$ does not necessarily have to be positive. $\mathrm{CCF}_{\text {ex }}$ rather refers to the next extremum when going forward in time (backward in $\tau$ ). At some positions, the reaction to the heat source occurs with reversed phase, i.e. at the time, that the heat source is switched on, the local temperature decreases. This happens, if the flow generated by the heat source changes the flow patterns in such a way, that more fresh air is brought to the respective position.

\section{Error analysis}

In order to quantify the accuracy of $\mathrm{CCF}_{\mathrm{ex}}$, we considered the uncertainties of the cross-correlation in (1) and the normalization factors in (2).

Prior to that, small (but constant) deviations of the actual from the nominal values for $q_{M}\left(<1.2 \%\right.$ ) and $\mathrm{P}_{\mathrm{H}}{ }^{\mathrm{m}}$ (maximum deviation $5.5 \%$, mean deviation $2.4 \%$ ) in the different flow scenarios, were compensated for by the employed normalization, see (2).

To estimate the remaining errors of the normalization factors, we took into account for each flow case the uncertainty of the reference signal autocorrelation $(0.2 \ldots 0.9 \%$, see below), the mean fluctuation (root mean square) of the heating power $(0.5 \ldots 1.9 \%)$, the measurement accuracy of the mass flow rate $q_{M}(2 \%)$, and the limited accuracy of the reference signal peak to peak value $(1 \%)$. These error sources add up by error propagation to about $2.4 \ldots 2.9 \%$ of $\mathrm{CCF}_{\mathrm{ex}}$, depending on the actual flow case.

The remaining noise in the reference signal, mentioned above, turned out to cancel out very efficiently in the autocorrelation function $(R \star R)(\tau)$. Considering the reference signal $R$ to consist of a periodic and a stochastic term, i.e. $R^{P}$ and $R^{N}$, the mixed terms, i.e. $R^{P} \star R^{N}$ cancel out in the autocorrelation, since the noise term is uncorrelated, and thus the relative error becomes:

$\frac{\Delta(R \star R)(0)}{(R \star R)(0)} \cong \frac{\left(R^{N} \star R^{N}\right)(0)}{\left(R^{P} \star R^{P}\right)(0)}$

For determination of this quantity we exploit the fact, that $R^{N} \star R^{N}$ is different from zero only in the vicinity of the origin, as $R^{N}$ decorrelates very fast.

Knowing that the maxima of the autocorrelation function $(R \star R)(\tau)$ would follow a straight line for a periodic signal, we determine $R^{N} \star R^{N}$ as the difference between $(R \star R)(0)$ and the intersection of the lines fitted to the other maxima of $(R \star R)(\tau)$ as depicted in Figs. 8 and 9.

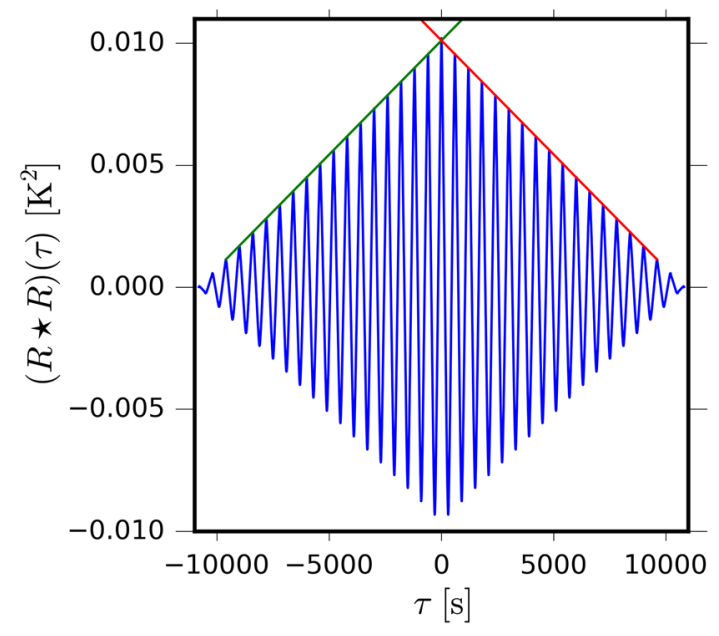

Fig. 8 Autocorrelation of the reference signal $R(t)$ for CCDV and $\mathrm{P}_{\mathrm{H}}=200 \mathrm{~W}$, full view

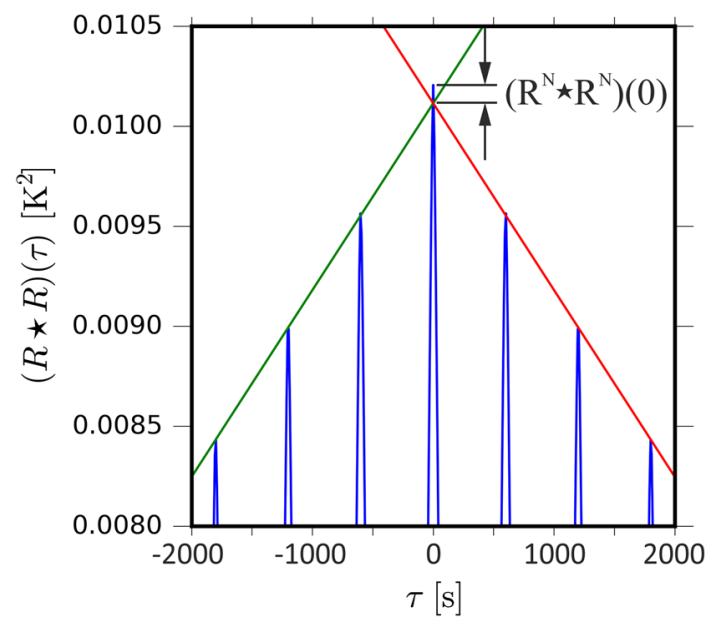

Fig. 9 Autocorrelation of the reference signal R(t) for CCDV and $P_{\mathrm{H}}=200 \mathrm{~W}$ in the vicinity of the first maximum

As a result, the relative error defined in (4) ranges from 0.2 through $0.9 \%$ in our measurements. If the mixed terms do not cancel out completely, some residual noise will be still present in the autocorrelation function $(R \star R)(\tau)$, observable as deviations of the maxima from the fitted lines. However, this remaining noise is negligible as compared to the error term defined by (4) in our reference signals.

With a similar procedure, the uncertainty during the determination of $\mathrm{CCF}_{\text {ex }}$ was quantified. The autocorrelation method described in Sect. 5 serves as a very efficient means to remove the uncorrelated noise from the signal, as demonstrated in Figs. 6 and 7. However, as opposed to the reference signal autocorrelation, only mixed noise terms occur in the cross-correlation. These should cancel out in the integrals, provided the integration time is long enough. 
However, some low-frequency fluctuations, as they are typical for cabin air flows, do not cancel out completely in CCF during our measurement interval. This results in the fact that the extrema in the cross-correlation function do not fall on a straight line as they would for a purely periodic temperature signal, see Fig. 10. Herewith, the main "noise" source in our experiment are the turbulent temperature fluctuations of the flow. The noise from the electronic circuitry is lower by a factor of about 50 and thus negligible. As we consider only temperature differences, the relative deviations of our signals due to the calibration uncertainties of the sensors are below two per mille and thus negligible too. To finally quantify the deterioration in determination of the first extremum, we located the first five extrema of CCF in each direction of $\tau$, and determined their (root mean square) deviation from a straight line, fitted to the data, as a measure for the uncertainty of $\mathrm{CCF}_{\mathrm{ex}}$.

The latter turned out to depend strongly on the actual measurement and flow conditions. To quantify this: the dynamic range, i.e. the ratio between maximum $\mathrm{CCF}_{\mathrm{ex}}$ and mean uncertainty, scales roughly with the heat source power and ranges between 140 and 660 for the presented data.

The total error, i.e. the combination of the normalization factor uncertainty and the uncertainty in the determination of the maximum in $\mathrm{CCF}_{\mathrm{ex}}$, using error propagation are depicted in the following 2D-plots. However, they are mostly not visible, because they are much smaller than the symbol size in most of the data.

It should be noted, though, that mixed convective flows, especially with buoyancy and inertia forces of equal strength, were sometimes found to exhibit unstable or oscillatory behavior, see e.g. [9, 15]. In such cases, small changes of the boundary conditions may lead to significant changes of larger flow structures. If present, such effects would, of course, surpass the pure measurement

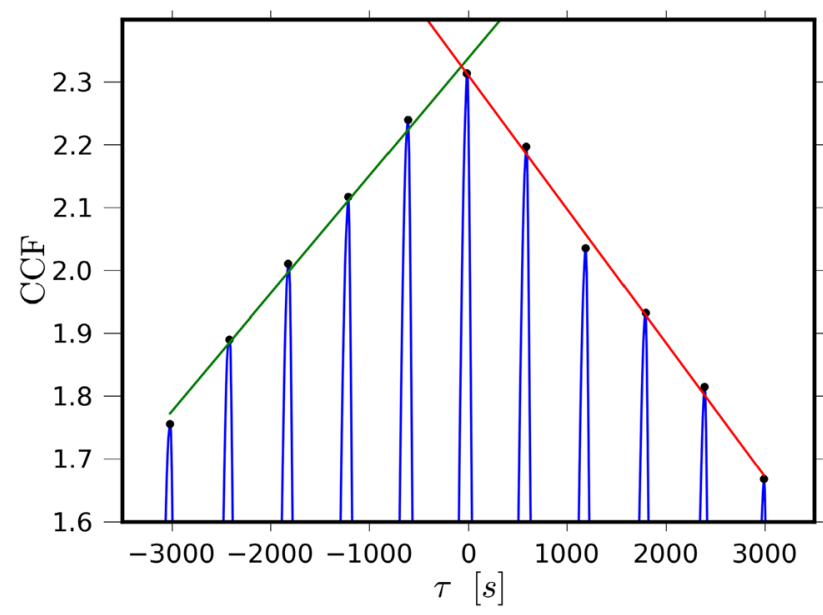

Fig. $10 \mathrm{CCF}$ for the sensor in row 9 of Fig. 7 with the local maxima of $\mathrm{CCF}$ and the linear fits to determine the uncertainty of $\mathrm{CCF}_{\mathrm{ex}}$ uncertainties quantified above. However, we did not observe such scenarios in the reported measurements.

\section{Experimental results}

Results for the two ventilation systems MV and CCDV are discussed in the following. Three settings of the local heat source are considered, see Table 1 . For the steady performance of the respective ventilation systems the reader is referred to [7].

\subsection{Mixing ventilation}

In order to analyze the propagation of the released heat in the cabin, we plotted the spatial distribution of $\mathrm{CCF}_{\mathrm{ex}}$ of the sensors at chest level in the passenger zone in Fig. 11 for the different heat source settings. The black iso-lines in Fig. 11a, b unveil, that at the two lower settings, the released heat remains located in the vicinity of the heat source (row 9). Propagation of heat in flight direction can be noticed only in the range of the seat rows adjacent to the source. Even inside of the row, where the source is placed (row 9), the heat remains strongly localized at the seats near the source. However, everything said so far refers to the selected sensor position (chest level). Knowing about the three
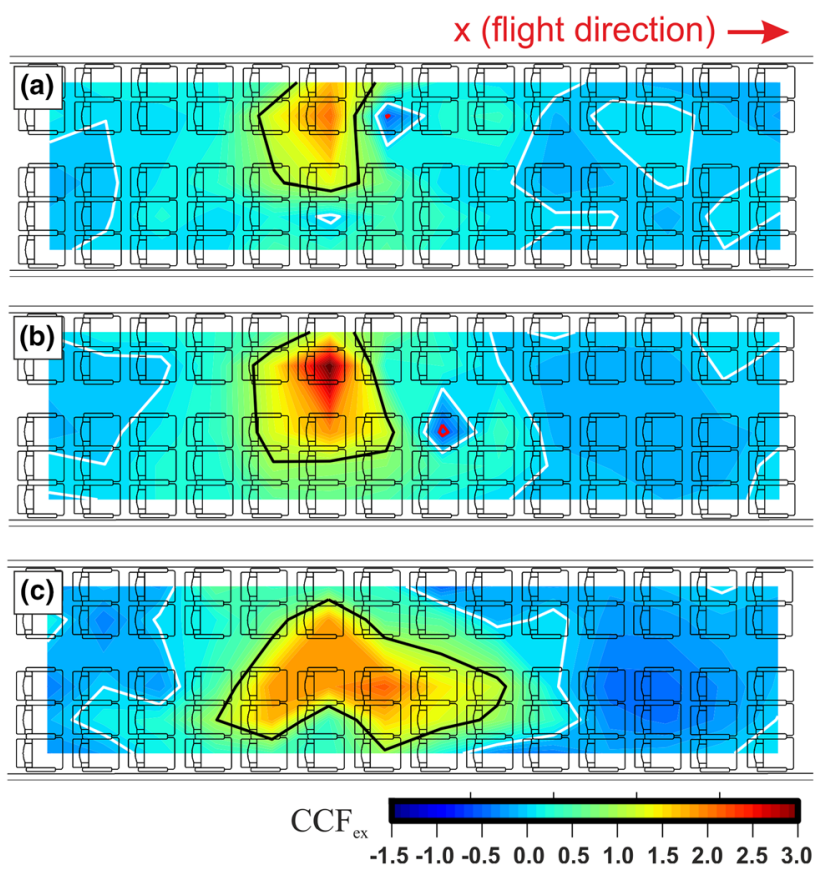

Fig. $11 \mathrm{CCF}_{\mathrm{ex}}$ of the temperatures measured for $\mathrm{MV}$ at chest level in the passenger zone as a function of seat position and depicted as contours for three nominal powers of the heat source: a $P_{\mathrm{H}}=100$ b $P_{\mathrm{H}}=200$ and c $P_{\mathrm{H}}=400 \mathrm{~W}$. The black, white and red lines indicate the 1.0, 0.0 and -0.5 iso-levels, respectively 


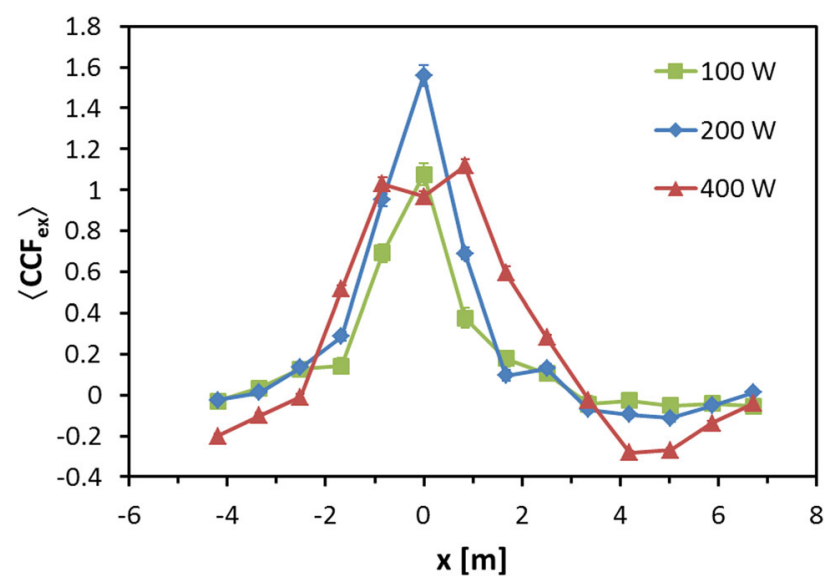

Fig. $12 \mathrm{CCF}_{\mathrm{ex}}$ of the temperatures measured for $\mathrm{MV}$ at chest level in the passenger zone, averaged over all seats in each row

dimensional nature of the cabin flows, other height levels might result in slightly different results. Nevertheless, switching to the highest source setting $\left(P_{\mathrm{H}}=400 \mathrm{~W}\right)$ in Fig. $11 \mathrm{c}$ induces a qualitative change of the temperature distribution. The source now clearly induces temperature changes in the next two to three adjacent seat rows in flight direction. Obviously, a change of the temperature from a predominantly passive scalar at the lower two settings to an active scalar, which is able to change the overall flow field in the cabin is observable in the data. In order to quantify the lateral extent of the impact of the heat source on the local temperatures, the seat-row average of $\mathrm{CCF}_{\mathrm{ex}}$ is given as a function of $\mathrm{x}$ in Fig. 12.

The propagation of the released heat in longitudinal cabin direction can be observed nicely herein. First, it shall be mentioned, that the maximal value is of the order of one for all three heat source settings, indicating, that

1. the released heat becomes diluted efficiently before the air enters the passenger zone, and

2. the temperature response near the source scales roughly with the heating power.

The spreading in $x$ direction amounts to about one seat row (plus / minus) for the lower settings and 2-3 rows for the highest setting.

In contrast to the results in the passenger zone, the values of $\mathrm{CCF}_{\mathrm{ex}}$ can be much larger in the aisle region, where the released heat is still concentrated, see Figs. 13 and 14. It is further observed, that the heat is far more localized in longitudinal direction as compared to the passenger zone, see Fig. 13.

Further insight in the propagation dynamics is obtained by the $\mathrm{CCF}_{\mathrm{ex}}$ values of the vertical RTD measurement rake in the aisle, see Fig. 14. The two lower settings result in a rather flat $\mathrm{CCF}_{\mathrm{ex}}$ distribution with a pronounced peak, indicating a lateral propagation of the released heat at a

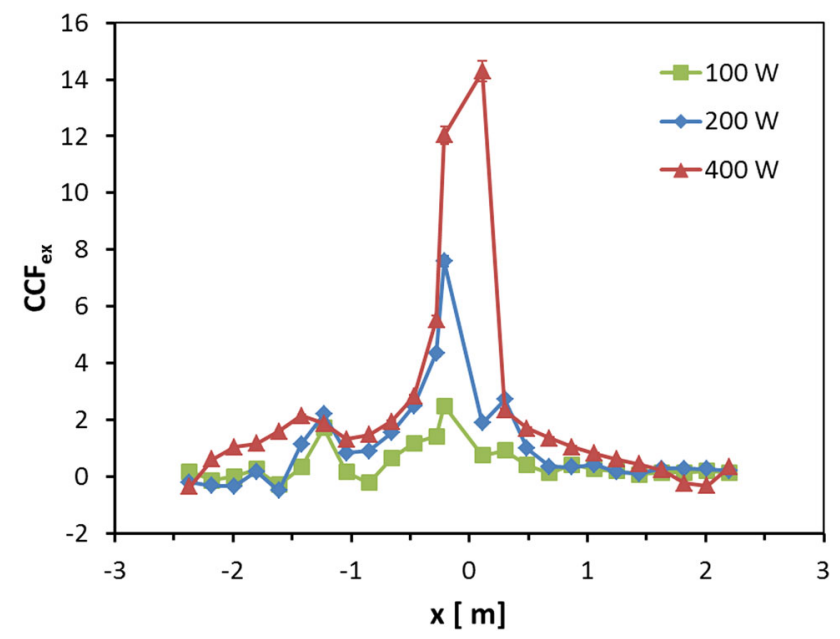

Fig. $13 \mathrm{CCF}_{\mathrm{ex}}$ of the temperatures measured for $\mathrm{MV}$ at chest level in the aisle using the horizontal RTD array for three nominal powers of the heat source

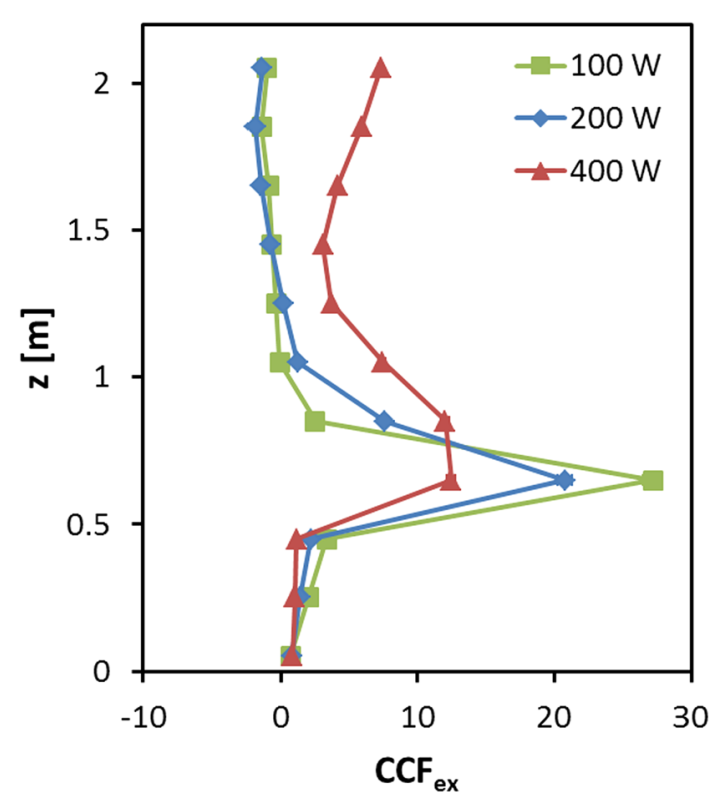

Fig. $14 \mathrm{CCF}_{\mathrm{ex}}$ of the temperatures measured for $\mathrm{MV}$ with the vertical RTD array in the aisle for three nominal powers of the heat source

height of $0.65 \mathrm{~m}$ due to the interaction between heat source and down welling fluid from the MV outlets in the aisle. Raising the source power from 100 to $200 \mathrm{~W}$ results in a significant increase of $\mathrm{CCF}_{\mathrm{ex}}$ at a level of 0.85 and $1.05 \mathrm{~m}$, which indicates a fortified rising of the warm air against the down welling fluid from the outlets. As the source power is further raised to $400 \mathrm{~W}$, clearly the upwelling of warm fluid can be observed in the $\mathrm{CCF}_{\mathrm{ex}}$ distribution over the whole aisle height, i.e. the buoyancy forces outweigh the inertia forces of the large scale circulations of the MV system. 


\subsection{Ceiling-based cabin displacement ventilation}

The contour plots of the $\mathrm{CCF}_{\mathrm{ex}}$ values at chest level for CCDV exhibit many similarities to those of MV, see Fig. 15. Again, at the two lower settings, the released heat remains located in the vicinity of the source. Propagation of heat in flight direction can be noticed in the seat rows adjacent to the heat source. The main difference, however, is, that regions with local minima of (negative) $\mathrm{CCF}_{\mathrm{ex}}$ values exist (see red iso-lines), which grow with increasing heating power. We think, that the resulting patterns with adjacent positive and negative $\mathrm{CCF}_{\mathrm{ex}}$ values, i.e. anti-correlated or opposite phased temperature behavior, indicate large scale circulations in the aisle region, which are induced by the heat source. Further, at the highest power, the spreading in flight direction does not increase as it was observed at MV (see also Fig. 16).

These observations are confirmed by the plot of $\mathrm{CCF}_{\mathrm{ex}}$ of the temperatures measured at chest level in the aisle, see Fig. 17.

Again, at least for the highest two powers, a dedicated peak near the heat source $(x=0)$ can be observed. It is adjoint by two side maxima, whose distance and amplitude rises with increasing source power. Farther away, the values tend to negative values. We think, that the cause for these structures is, that the released heat already rises to the CCDV
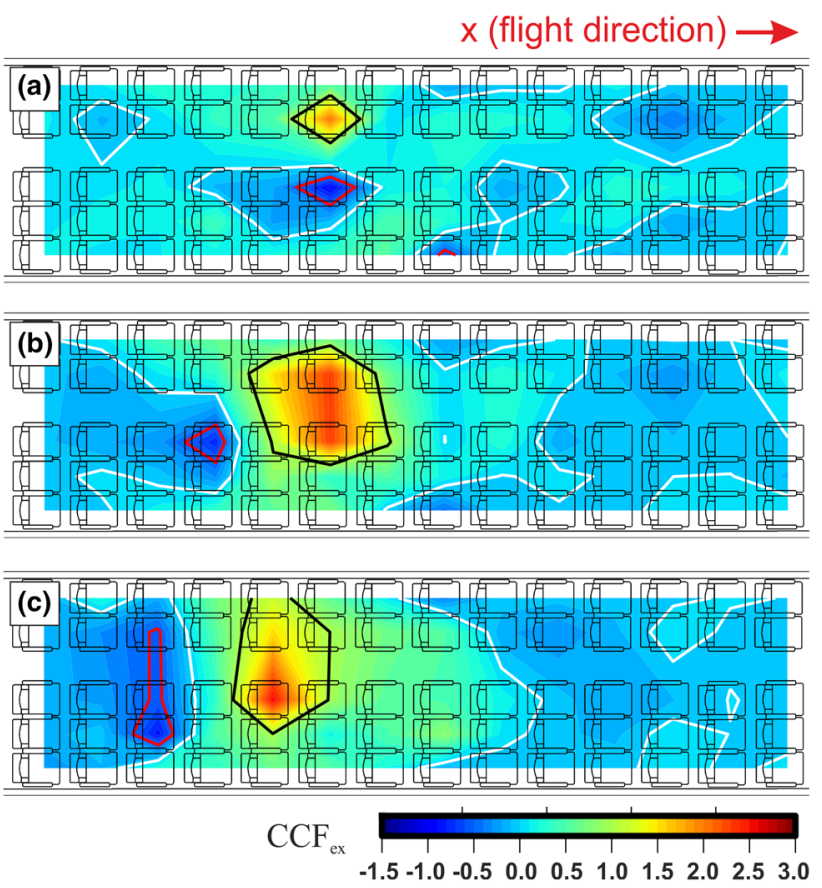

Fig. $15 \mathrm{CCF}_{\mathrm{ex}}$ of the temperatures measured for $\mathrm{CCDV}$ at chest level in the passenger zone as a function of seat position, depicted as contours for three nominal powers of the heat source: a $P_{\mathrm{H}}=100$ b $P_{\mathrm{H}}=200$ and c $P_{\mathrm{H}}=400 \mathrm{~W}$. The black, white and red lines indicate the 1.0, 0.0 and -0.5 iso-levels, respectively

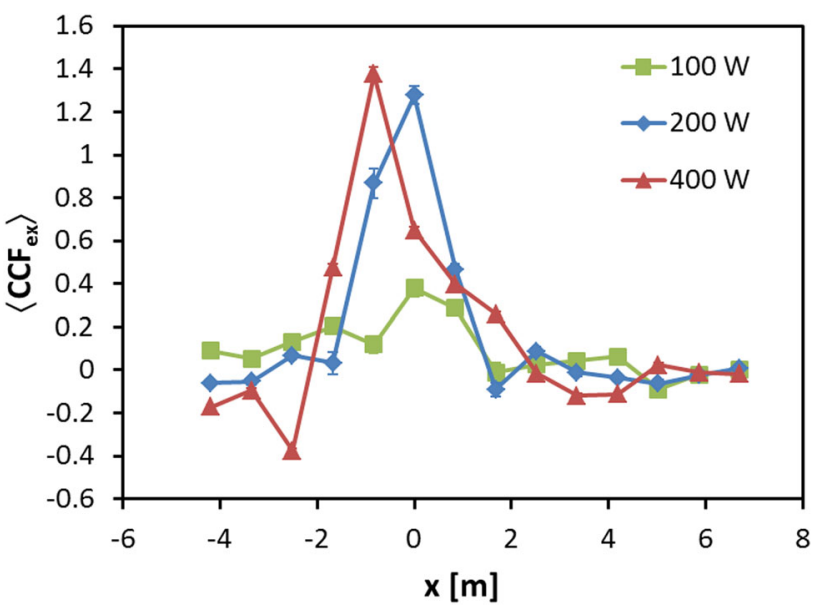

Fig. $16 \mathrm{CCF}_{\mathrm{ex}}$ of the temperatures measured for CCDV at chest level in the passenger zone, averaged over all seats in each row

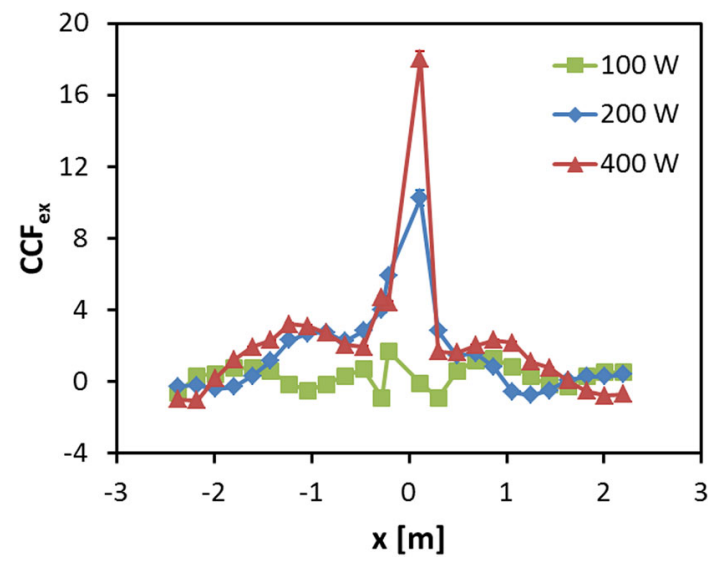

Fig. $17 \mathrm{CCF}_{\mathrm{ex}}$ of the temperatures measured for CCDV at chest level in the aisle with the horizontal RTD array at three nominal powers of the heat source

outlets at source powers of $200 \mathrm{~W}$, where it dilutes with fresh air, but also laterally displaces a significant amount of the fresh air flow. As a consequence, the slightly diluted, warm air descends alongside of the heat source, causing the side peaks of $\mathrm{CCF}_{\text {ex }}$. Beyond these, even more fresh air descends, which causes the negative $\mathrm{CCF}_{\mathrm{ex}}$ values with the corresponding out-of-phase behavior. As the source power increases, the upwelling volume flow induced by the heat source increases, and so does the displacement of the fresh air at the ceiling. Consequently, the wavelength of the observed structures in longitudinal cabin direction increases. This behavior is indicated as well by Fig. 18, which shows, that already at a power of $200 \mathrm{~W}$, warm air near the heat source can rise up to the ceiling due to the lower inertia forces at CCDV as compared to MV.

At the lowest power, a peak at $0.65 \mathrm{~m}$ again indicates a lateral propagation of the warm air, governed by the down 


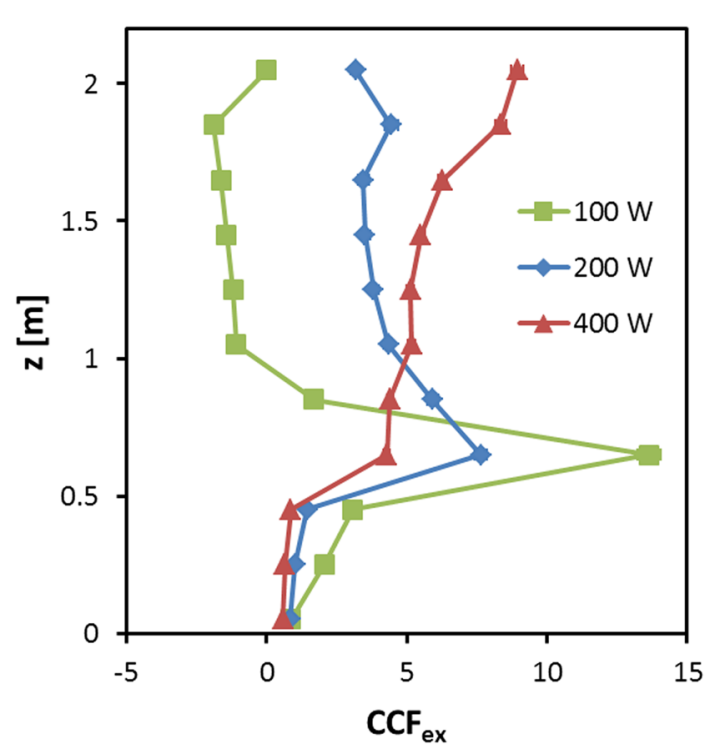

Fig. $18 \mathrm{CCF}_{\text {ex }}$ of the temperatures measured for CCDV with the vertical RTD array in the aisle at three nominal powers of the heat source

welling fluid in the aisle. As opposed to that, at the highest power, the $\mathrm{CCF}_{\mathrm{ex}}$ values monotonically rise from floor to ceiling. In the plot of the row-wise average of the absolute $\mathrm{CCF}_{\mathrm{ex}}$ values (Fig. 16), we can observe that, in contrast to $\mathrm{MV}$, the peak position is not fixed to the position of the heat source, but is rather shifted by one row to the rear at the highest source power. Further, at the highest source power, the spreading of heat in $x$-direction is much smaller at CCDV as compared to MV, see Figs. 12 and 16. It seems that spreading of the released heat is restricted to the aisle region and much less pronounced in the passenger zone.

\section{Conclusions}

For the first time, the propagation of localized heat loads in aircraft cabin air flow was investigated. Two ventilation systems, i.e. mixing ventilation and ceiling-based cabin displacement ventilation have been studied experimentally in the Do 728 test facility of the German Aerospace Center in Göttingen. A heat source with a reaction time of a fraction of the nominal air exchange time was built and placed inside the cabin to study the response functions of the cabin temperatures to the released heat. It was heated periodically by means of a programmable power supply. The impact on the cabin temperatures was quantified by evaluating the first extremum of the cross-correlation function between the local temperatures and the normalized surface temperature of the heat source, $\mathrm{CCF}_{\mathrm{ex}}$. Large values for $\mathrm{CCF}_{\mathrm{ex}}$ are found in the aisle region, where the heat is released, for both ventilation systems. However, the heat gets mixed up very quickly in MV and CCDV, resulting in moderate $\mathrm{CCF}_{\mathrm{ex}}$ values in the passenger zone. The values are of comparable magnitude for MV and CCDV and scale in a first approximation with the heating power. The response to the heat source is strongly localized in the passenger zone at the lower two power settings of the source. Mainly the seat rows before and after the one where the source is placed are affected. At the highest setting, the impact of the heat load is spread much farther in the passenger zone at MV, while it is seemingly still concentrated at CCDV. Measurements with the RTD-rakes in the aisle reveal, that the buoyancy forces outweigh the inertia forces at a nominal power of $400 \mathrm{~W}$ for MV, while this is already the case in CCDV at a power of $200 \mathrm{~W}$. Rising the heating power from 200 to $400 \mathrm{~W}$ in MV results in an increased spreading of the impact in the passenger zone, while the heat is strongly localized in the aisle. At CCDV, the heat becomes distributed farther in longitudinal direction in the aisle, while it remains more localized in the passenger zone. Clearly, the different air-guiding principles of the momentum-driven MV and the buoyancy-driven CCDV imply different propagation paths and dynamics of locally released heat loads. At CCDV, we observed the fingerprints of large-scale structures in longitudinal aisle direction, which help to dilute the released heat efficiently.

Further investigations addressing hybrid ventilation settings, evaluation of further measurement positions and flow visualization are required to shed more light on the underlying, complex transport processes.

Acknowledgments This project was supported by the German Federal Ministry of Economics and Energy under Grant Number 20K1101E on the basis of a decision of the German Bundestag. The responsibility of the content is the authors.

\section{References}

1. Knepple, R.A.: Die energie-autarke Kabine-das Ende der klassischen Bordnetze, Deutscher Luft- und Raumfahrtkongress, Berlin, Germany, 10-12 September 2012

2. Kühn, M., Bosbach, J., Wagner, C.: Experimental parametric study of forced and mixed convection in a passenger aircraft cabin mock-up. Build. Environ. 44(5), 961-970 (2009)

3. Schmid, M., Müller, D., Gores, I., Markwart, M.: Numerical study of different air distribution systems for aircraft cabins, 11th international conference on indoor air quality and climate, Copenhagen, Denmark, 17-22 August 2008

4. Yin, S., Zhang, T.A., New under-aisle displacement air distribution system for wide-body aircraft cabins, eleventh international IBPSA conference, Glasgow, Scotland, 1030-1036 2009

5. Bosbach, J., Heider, A., Dehne, T., Markwart, M., Gores, I., Bendfeldt, P.: Evaluation of cabin displacement ventilation under flight conditions, ICAS2012, Brisbane, Australia, 23-28 September 2012, paper 304

6. Zhang, T., Chen, Q.: Novel air distribution systems for commercial aircraft cabins. Build. Environ. 42(4), 1675-1684 (2007) 
7. Bosbach, J., Lange, S., Dehne, T., Lauenroth, G., Hesselbach, F., Allzeit, M.: Alternative ventilation concepts for aircraft cabins. CEAS Aeronaut. J. 4, 301-313 (2013)

8. Mangili, A., Gendreau, M.A.: Transmission of infectious diseases during commercial air travel. Lancet 365, 989-996 (2005)

9. Kühn, M., Bosbach, J., Wagner, C.: Stability of the air flow in a two aisle cabin model, first CEAS European air and space conference, Berlin, Germany, 10-13 September 2007

10. Bosbach, J., Heider, A., Dehne, T., Markwart, M., Gores, I., Bendfeldt, P.: Flight testing of alternative ventilation systems for aircraft cabins. In: New Results in Numerical and Experimental Fluid Mechanics IX Notes on Numerical Fluid Mechanics and Multidisciplinary Design, p. 124 Springer, Cham Heidelberg New York Dordrecht London. Seiten 275-283. ISBN 978-3-31903157-6. ISSN 1612-2909

11. Bianco, V., Manca, O., Nardini, S., Roma, M.: Numerical investigation of transient thermal and fluiddynamic fields in an executive aircraft cabin. Appl. Therm. Eng. 29, 3418-3425 (2009)

12. Yan, W., Zhang, Y., Sun, Y., Li, D.: Experimental and CFD study of unsteady airborne pollutant transport within an aircraft cabin mock-up. Build. Environ. 44, 34-43 (2009)

13. Mazdumar, S., Poussou, S.B., Lin, C.H., Isukapalli, S.S., Plesniak, M.W., Chen, Q.: Impact of scaling and body movement on contaminant transport in airliner cabins. Atmos. Environ. 45, 6019-6028 (2011)

14. Wu, C., Ahmend, N.A.: Numerical study of transient aircraft cabin flowfield with unsteady air supply. J. Aircr. 48, 1994-2000 (2011)

15. Schmeling, D., Bosbach, J., Wagner, C.: Oscillations of the largescale circulation in turbulent mixed convection in a closed rectangular cavity. Exp. Fluids 54, 1517 (2013) 